Editorial

\title{
Mechanism and Therapies of Oxidative Stress-Mediated Cell Death in Ischemia Reperfusion Injury
}

\author{
Haobo Li $\mathbb{D},{ }^{1,2}$ Zhengyuan Xia $\mathbb{D}^{1},{ }^{1}$ Yanfang Chen $\mathbb{D},{ }^{3}$ Dake $Q i \mathbb{D}^{4},{ }^{4}$ and Hong Zheng ${ }^{5}$ \\ ${ }^{1}$ The University of Hong Kong, Pok Fu Lam, Hong Kong \\ ${ }^{2}$ Harvard Medical School, Harvard University, Boston, MA, USA \\ ${ }^{3}$ Wright State University Boonshoft School of Medicine, Dayton, OH, USA \\ ${ }^{4}$ Memorial University of Newfoundland, St. John's, NL, Canada \\ ${ }^{5}$ The First Affiliated Hospital of Xinjiang Medical University, Xinjiang, China \\ Correspondence should be addressed to Haobo Li; haobo.li@mgh.harvard.edu
}

Received 15 October 2017; Accepted 16 October 2017; Published 24 June 2018

Copyright ( $\odot 2018$ Haobo Li et al. This is an open access article distributed under the Creative Commons Attribution License, which permits unrestricted use, distribution, and reproduction in any medium, provided the original work is properly cited.

Ischemia reperfusion-induced tissue injuries and organ failure represent the major causes of postoperative mortality and morbidity. Oxidative stress-mediated cell death plays a vital role in this pathology [1].

Induction of different types of cell death (e.g., apoptosis, necroptosis, and pyroptosis) triggered by reactive oxygen species (ROS) plays an important role in ischemia reperfusion injury (IRI) in multiple organs [2]. In this special issue, Z. Qiu et al. reported that, under hyperglycemic conditions, induction of nod-like receptor protein 3 (NLRP3) inflammasome-mediated pyroptotic cell death is critical in myocardial IRI, while inhibition of the inflammasome with specific inhibitors or ROS scavengers, $\mathrm{N}$-acetylcysteine, reduced pyroptotic cell death and attenuated myocardial IRI. Moreover, oxidative stress-induced apoptotic and necroptotic cell deaths also play important roles in cardiac dysfunction as reported in this special issue by S. Peng et al. and $\mathrm{N}$. Zeng et al, which showed that oxidative stress by increasing myocardial cell apoptosis and necroptosis leads to cardiac dysfunction in septic rats. Treatment with either PPAR- $\gamma$ or brain-derived neurotrophic factor could reduce such types of cell death and attenuate cardiac dysfunction by reducing oxidative stress.

Reperfusion-induced oxidative stress is the major contributor in IRI. Thus, therapies that increase antioxidant capacity may protect organs against IRI [3]. Through its antioxidant capacity, the intravenous anesthetic propofol has been shown to alleviate myocardial IRI in patients undergoing cardiac surgery $[4,5]$ and in animals subjected to myocardial and intestinal IRI $[6,7]$. In this special issue, F. Deng et al. reported that pretreatment with propofol to inhibit caveolae suppressed microvesicle release and attenuated cardiomyocyte hypoxia reoxygenation injury. Further, H.-J. Su et al. reported in this special issue that propofol conditioning confers antioxidative and cardioprotective effects against myocardial IRI through enhancing endogenous endocannabinoid release and the subsequent activation of CB2 receptor signaling. On the other hand, Z. Liu et al. reported that simvastatin pretreatment in donors may reduce hepatic oxidative stress through a fruppel-like factor 2dependent mechanism which attenuated hepatic liver IRI and improved liver function recovery in rats that underwent liver transplantation. X.-T. Yan et al showed that treatment with PEP-1-heme oxgenase-1 fusion protein confers protection against septic shock-induced lung injury by reducing hepatic oxidative stress and inflammation, likely through suppression of toll-like receptor- 4 and NF- $\kappa \mathrm{B}$.

Aggravated inflammation, which has been shown to subsequently induce oxidative stress, has been proposed as a major cause of IRI and organ injury [8]. Attempts to attenuate organ injury by solely decreasing inflammation or increasing antioxidant capacity have achieved limited success $[9,10]$, indicating that multifaceted therapies combining anti-inflammatory and antioxidant approaches may be 
necessary for effective treatment. In this special issue, Q. Shan et al. reported that ingestion of $2,2^{\prime}, 4,4^{\prime}$-tetrabromodiphenyl ether (BDE-47), one of many persistent organic pollutants, leads to oxidative injury and cell death in the kidney by inducing the NLRP3 inflammasome. They further showed that application of troxerutin, a flavonoid with pharmacological antioxidant and anti-inflammatory activity, reduced BDE-47-indcued oxidative stress and cytotoxicity in the kidney through CXCR4/TXNIP/NLRP3 and Nrf2 signaling pathways. The findings of $\mathrm{Q}$. Shan et al. may promote further in-depth studies regarding the interaction of inflammation and oxidative stress in the setting of IRI, which may facilitate our understanding of the pathophysiology of IRI and the development of new therapies for the disease.

We hope that the research articles presented in this special issue contribute to the understanding of current advancements and the mechanisms of oxidative stressmediated cell death in ischemia reperfusion injury. It is also our hope to stimulate further efforts in the investigation of the pathology of ischemia reperfusion injury and the development of therapy for the disease.

\section{Acknowledgments}

We would like to thank all of the authors for submitting their exciting research for consideration for publication, and we also thank all of the reviewers for sharing their expertise and precious time in reviewing the manuscripts. Thanks to all of those who contributed to the success of this special issue.

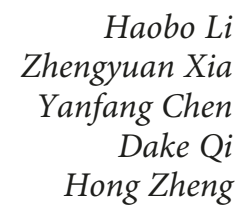

\section{References}

[1] G. A. Kurian, R. Rajagopal, S. Vedantham, and M. Rajesh, "The role of oxidative stress in myocardial ischemia and reperfusion injury and remodeling: revisited," Oxidative Medicine and Cellular Longevity, vol. 2016, Article ID 1656450, 14 pages, 2016.

[2] L. Minutoli, D. Puzzolo, M. Rinaldi et al., "ROS-mediated NLRP3 inflammasome activation in brain, heart, kidney, and testis ischemia/reperfusion injury," Oxidative Medicine and Cellular Longevity, vol. 2016, Article ID 2183026, 10 pages, 2016.

[3] H. Li, W. Yao, M. G. Irwin et al., "Adiponectin ameliorates hyperglycemia-induced cardiac hypertrophy and dysfunction by concomitantly activating Nrf2 and Brg1," Free Radical Biology and Medicine, vol. 84, pp. 311-321, 2015.

[4] Z. Xia, Z. Huang, and D. M. Ansley, "Large-dose propofol during cardiopulmonary bypass decreases biochemical markers of myocardial injury in coronary surgery patients: a comparison with isoflurane," Anesthesia \& Analgesia, vol. 103, no. 3, pp. 527-532, 2006.

[5] D. M. Ansley, K. Raedschelders, P. T. Choi, B. Wang, R. C. Cook, and D. D. Chen, "Propofol cardioprotection for on- pump aortocoronary bypass surgery in patients with type 2 diabetes mellitus (PRO-TECT II): a phase 2 randomizedcontrolled trial," Canadian Journal of Anesthesia/Journal canadien d'anesthésie, vol. 63, no. 4, pp. 442-453, 2016.

[6] X. Gan, D. Xing, G. Su et al., "Propofol attenuates small intestinal ischemia reperfusion injury through inhibiting NADPH oxidase mediated mast cell activation," Oxidative Medicine and Cellular Longevity, vol. 2015, Article ID 167014, 15 pages, 2015.

[7] Z. Xia, D. V. Godin, and D. M. Ansley, "Application of highdose propofol during ischemia improves postischemic function of rat hearts: effects on tissue antioxidant capacity," Canadian Journal of Physiology and Pharmacology, vol. 82, no. 10, pp. 919-926, 2004.

[8] W. Yao, H. Li, G. Luo et al., "SERPINB1 ameliorates acute lung injury in liver transplantation through ERK1/2-mediated STAT3-dependent HO-1 induction," Free Radical Biology and Medicine, vol. 108, pp. 542-553, 2017.

[9] M. F. Tsan, "Superoxide dismutase and pulmonary oxygen toxicity: lessons from transgenic and knockout mice (review)," International Journal of Molecular Medicine, vol. 7, no. 1, pp. 13-22, 2001.

[10] R. J. van Klaveren, D. Dinsdale, J. L. Pype, M. Demedts, and B. Nemery, "N-acetylcysteine does not protect against type II cell injury after prolonged exposure to hyperoxia in rats," American Journal of Physiology Lung Cellular and Molecular Physiology, vol. 273, no. 3, pp. L548-L555, 1997. 


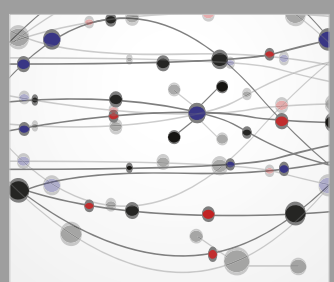

The Scientific World Journal
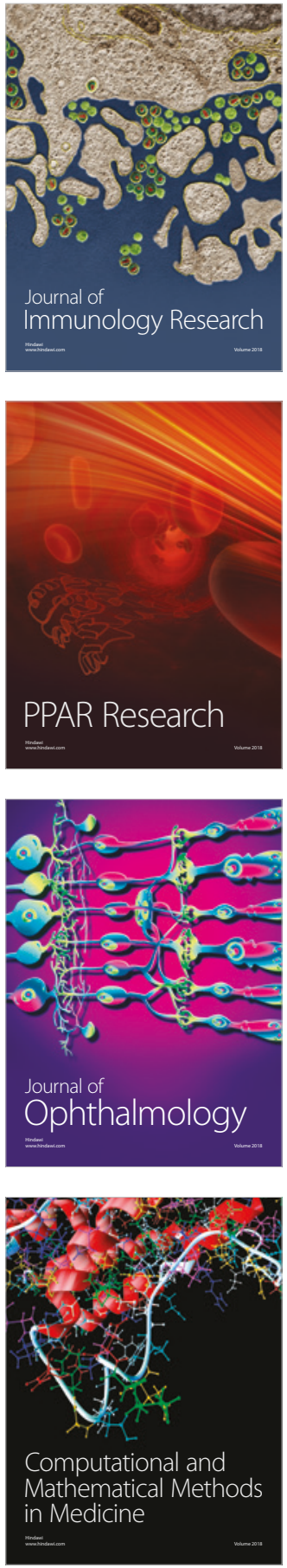

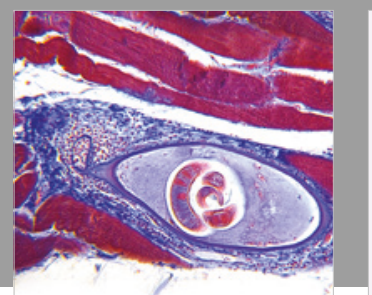

Gastroenterology Research and Practice

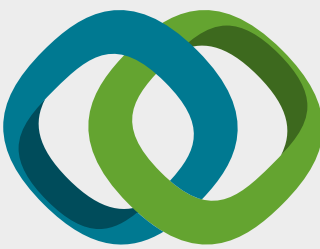

\section{Hindawi}

Submit your manuscripts at

www.hindawi.com
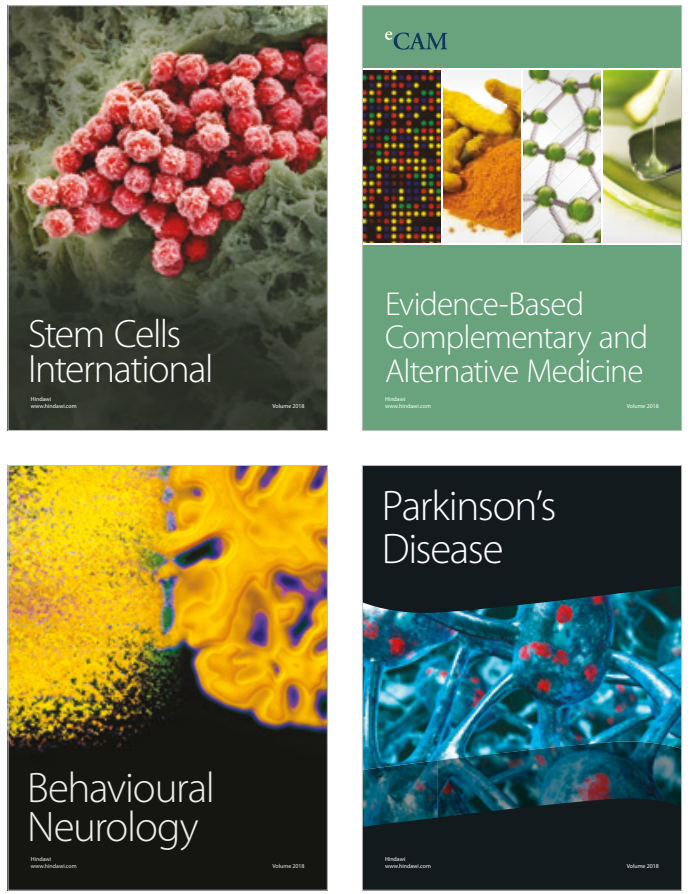

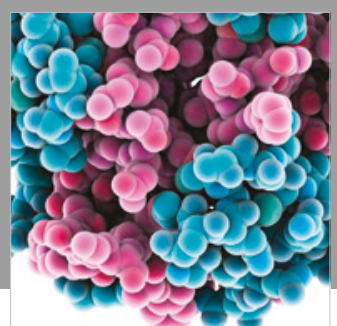

ournal of

Diabetes Research

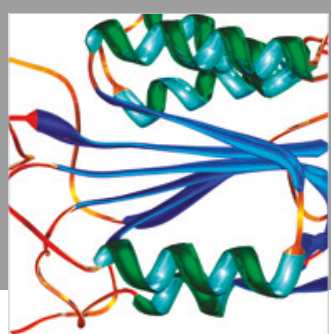

Disease Markers
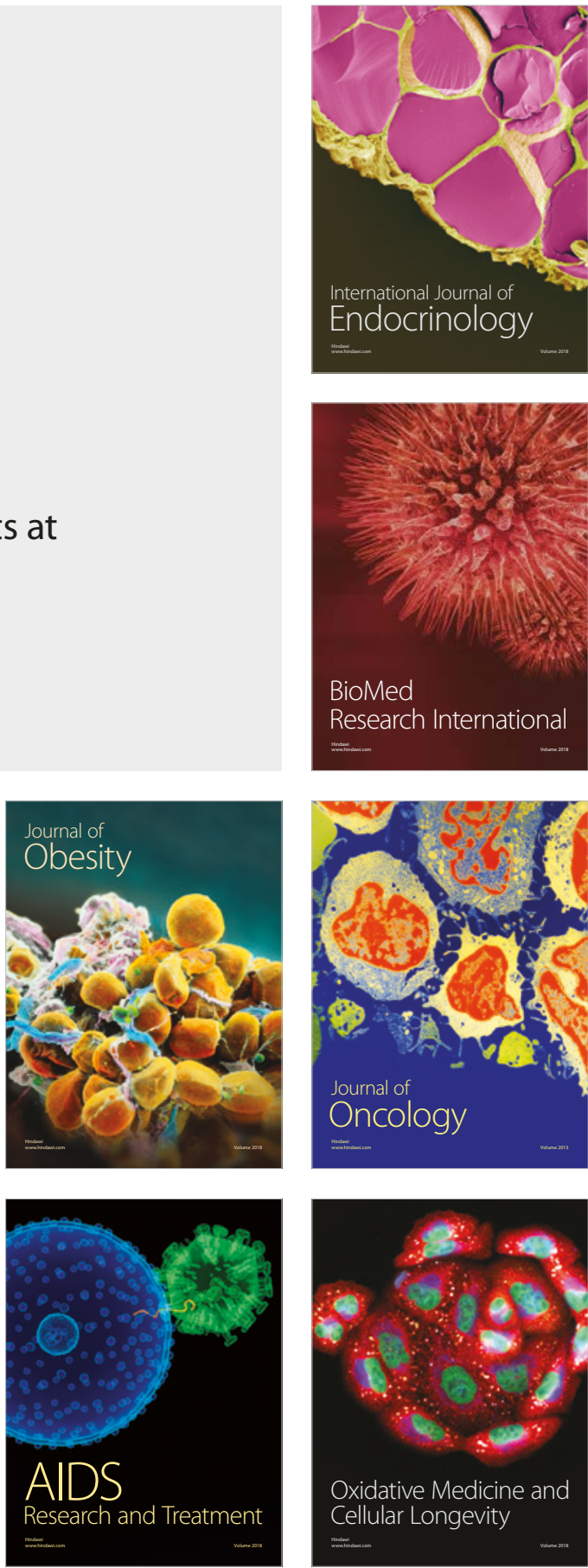